\title{
Bromocriptine in Parkinson's disease: a double-blind study comparing "low-slow" and "high-fast" introductory dosage regimens in de novo patients
}

\author{
From the UK BROMOCRIPTINE RESEARCH GROUP*
}

SUMMARY One hundred and thirty four patients with previously untreated Parkinson's disease were recruited to a multicentre double blind study comparing two introductory dosage regimens of bromocriptine: a low/slow regimen increasing to a maximum of $25 \mathrm{mg} /$ day and a high/fast regimen increasing to a maximum of $100 \mathrm{mg} /$ day over a 26 week period. It was hoped to determine the minimum dose of bromocriptine required with either regimen. A patient's ability to achieve a $33 \%$ improvement in clinical rating scores was recorded using a combined score of functional disability (Webster) and self rated daily living activities (ADL). Sixty five out of 129 patients $(50 \%)$ had reached the improvement criteria in 26 weeks, 37 (49\%) from the "slow" and $28(53 \%)$ from the "fast" group (N.S.). However, if only those patients still in the trial at the end of 26 weeks are considered the relevant percentages are $73 \%$ and $88 \%(p<0.05)$. Statistical analysis allowing for censored observations was used to examine group differences in dosage and time at improvement. This excluded the patients who had dropped out due to side effects from the calculations. The results indicated a marked difference between groups in both dose (slow $22.0 \mathrm{mg}$, fast $55.4 \mathrm{mg}(\mathrm{p}<0.01$ ) and time (slow 22.8 weeks and fast 14.4 weeks $(\mathrm{p}<0.05)$. Severe side effects necessitated withdrawal from the trial in 34 patients, a larger proportion $19(36 \%)$ being in the fast group compared with $15(20 \%)$ from the slow group $(p<0.05)$. The number of dropouts due to non effect was $2(4 \%)$ in the fast and $10(13 \%)$ in the slow. The only predisposing factor relating to dropping out due to side effects was a high initial ADL $(p<0.01)$. It is concluded that bromocriptine is an effective de novo treatment for Parkinson's disease. The "fast" introductory regimen is less well tolerated than the "slow", but the latter has the disadvantage of a long delay before patients reach an effective dose. It is recommended that it would be wise to adopt an intermediate dosing strategy.

Bromocriptine (Parlodel), a semisynthetic centrally acting dopamine agonist, has been used in the treatment of Parkinson's disease since 1974. ' Most authors have concerned themselves with its use as adjuvant treatment for patients already receiving levodopa, particularly those experiencing long term complications of treatment. ${ }^{2-6}$ Its role in previously untreated patients is less clear. Several studies of bromocriptine as sole therapy have been reported. ${ }^{7-11}$ The mean daily

*B S Everitt, D Hand (statisticians), M A Barrie, D Bates, H G Boddie, J D Carroll, L Cleeves, P G Cleland, D W Davidson, L J Findley, M Gibson, R B Godwin-Austen, J Gumpert, R Hardie, E Hay, B R Kendall, C Kennard, A J Lees, D L McClellan, I D Melville, P Millac, R P Murphy, P K Newman, D M Park, I Pye, D Riddoch, P Savundra, G M Stern, M Swallow.

Address for reprint requests: Dr H G Boddie, Department of Neurology, North Staffordshire Royal Infirmary, Princes Road, Hartshill, Stoke-on-Trent, SR4 7LN, UK.

Received 19 October 1987 and in final revised form 31 August 1988. Accepted 6 September 1988 dose used in these studies ranged from $25.5 \mathrm{mg}$ to 70 $\mathrm{mg} /$ day. In 1981 Teychenne," suggested that lower doses (mean dose $<14 \mathrm{mg} /$ day) might in this group of patients give a similar initial benefit to that obtained with high dose treatment.

Bromocriptine used alone is less likely than levodopa to produce certain features of late failure of control of the disease, such as drug induced dyskinesia and dystonia.

Rinne $^{6}$ reported that two out of $21(10 \%)$ of his patients treated with bromocriptine alone for three years developed peak dose dyskinesia, whereas comparable results in a levodopa treated group reported by the same author was $121 / 217(57 \%)$. Rascol ${ }^{8}$ also reported a low incidence of dyskinesia, four out of 44 patients $(10 \%)$ treated either alone with bromocriptine or in combination with levodopa. Lees and Stern' followed a group of 50 patients initially on bromocriptine alone or subsequently switched to levodopa based treatment. After one year 28 patients were still receiv- 
ing bromocriptine therapy. Of these one patient developed peak dose dyskinesia, a considerably lower incidence than in a retrospective control group matched for age at onset of disease, disease severity, and duration of disease prior to treatment, in which the corresponding figure for dyskinesia was 19/29. These authors subsequently found at the end of three years that no other patients on bromocriptine alone had end of dose dyskinesia, the on-off phenomenon or end of dose deterioration. The authors suggest that levodopa may be partially responsible for lowering the threshold to drug induced dyskinesias. The higher incidence of end of dose deterioration in the levodopa treated group (35\%) may be attributed to a progressive inability of dopaminergic systems to convert dopa to dopamine. No patient treated with bromocriptine as the sole dopaminergic treatment has been observed to develop on-off swings.

We report here the results of the first 26 weeks of a multicentre trial, conducted in 18 neurology departments in the UK in which two introductory dosage regimens "high/fast" and "low/slow" were compared on a double blind basis, the former based on established practice ${ }^{8-10}$ and the latter as proposed by Teychenne." The aims of the trial were threefold. Firstly, it was intended to determine the minimum effective dose of bromocriptine. Secondly, it was hoped to establish the optimum rate of introduction of bromocriptine and thirdly, the tolerance and quality of the response of patients to bromocriptine was monitored over the 26 week period.

Incremental dosing continued until a patient was satisfied that he could cope with his disabilities and his clinical rating score improved by one third. Subsequently, patients will be followed for 5 years to establish whether their initial response to bromocriptine is maintained, or to what extent their bromocriptine dose has to be increased. The long term progress of these patients and that of both early and late treatment failures who have transferred to levodopa based treatment will be reported later.

\section{Patients and methods}

\section{Trial design}

Initially patients were randomly allocated under double blind conditions, for up to 26 weeks to one of the two bromocriptine treatment regimens the "low/slow" or "high/fast". The low/slow provided doses of bromocriptine up to $25 \mathrm{mg}$ and the high/fast doses up to $100 \mathrm{mg}$. The randomisation code provided for $60 \%$ of patients to be randomised to the slow regimen and $40 \%$ allocated to the fast regimen, there being considerable evidence in the literature on the latter regimen. ${ }^{79}{ }^{10}$ It was hoped to obtain more information on the slow regimen. Recruitment to the trial was 80 to the slow and 54 to the fast group. This only reduced the power of the study by $2 \% .^{20}$
Table 1 Clinical weighting scales

\begin{tabular}{|c|c|c|}
\hline & Score & $\begin{array}{c}\text { Score } \times \\
\text { Weighting weighting }\end{array}$ \\
\hline $\begin{array}{l}\text { Self-rated score for } \\
\text { activities of daily } \\
\text { living (ADL): }\end{array}$ & $\begin{array}{l}\text { Dressing } \\
\text { Eating } \\
\text { Food preparation } \\
\text { Cleaning/tidying } \\
\text { home } \\
\text { Getting out of bed } \\
\text { Turning in bed } \\
\text { Getting out of chair } \\
\text { Climbing stairs } \\
\text { Use of toilet } \\
\text { Use of hand tools } \\
\text { Bathing } \\
\text { Shopping and use of } \\
\text { local transport } \\
\text { SUB TOTAL }\end{array}$ & $\begin{array}{l}4 \\
4 \\
2 \\
3 \\
\\
3 \\
4 \\
4 \\
2 \\
5 \\
2 \\
4 \\
3\end{array}$ \\
\hline Webster scale items: & $\begin{array}{l}\text { Bradykinesia and } \\
\text { handwriting } \\
\text { Rigidity } \\
\text { Posture } \\
\text { Upper extremity } \\
\text { swing } \\
\text { Gait } \\
\text { Tremor } \\
\text { Facies } \\
\text { Seborrhoea } \\
\text { Speech } \\
\text { Self care } \\
\text { SUB TOTAL }\end{array}$ & $\begin{array}{r}11 \\
8 \\
8 \\
1 \\
12 \\
8 \\
1 \\
1 \\
10 \\
0\end{array}$ \\
\hline
\end{tabular}

Subjects

One hundred and thirty four patients (82 male, 52 female) consented to participate in the trial. Anticholinergic drugs or amantidine were the only other drugs previously used in theo treatment of their Parkinson's disease and such treatment had been optimised and the dose held constant for a minimum of 2 weeks. All were suffering from mild to? moderately severe stable Parkinson's disease where an average of two clinical ratings (see table 1), performed on separate days before entry, was between 50 and 150 . The maximum theoretical disability score which was possible was 300.

Amongst other reasons that patients were excluded from the trial were if they had a life expectancy of less than five years, if they suffered from an additional disorder which would interfere with their clinical rating, if they were taking monoamine oxidase inhibitor drugs or had experienced psychotic illness or a marked degree of dementia.

The nature of the trial was explained to the patient, where possible with a relative present. The trial protocol was approved by the respective hospital Ethical Committees.

\section{Clinical rating}

The time interval from onset of symptoms of Parkinson's disease to entry to the trial was recorded together with any past history of depressive illness. The pattern of the patient's current disability was recorded using individual items from the Webster scale ${ }^{16}$ and a self-rated scale for activities of daily living (ADL). These were repeated on two separate days.

Thereafter, clinic visits were at intervals of not more than three weeks if dosage had been adjusted. At each visit the Webster scale ADL and daily bromocriptine dose were 
recorded, along with any other relevant comments related to drug dosage, patient's general health, response to treatment and the presence or absence of side effects.

The Webster rating scale was chosen as a well known, commonly used basis for clinical rating. To improve the extent to which the Webster score reflects the patient's disability, the scale was weighted, emphasis being given to those items of the scale which, if affected by Parkinson's disease were likely to cause functional disability. Additionally, the self care items were also weighted (wADL) to rate individually those activities of daily living which were required of slightly to moderately disabled persons. More weight was given to Webster scale items (60) than to ADL (40). All items were scored from $0-3$ and the raw Webster score and total clinical rating score noted. The detailed scales are shown in table 1 .

\section{Treatment}

The trial allowed for two double blind randomised treatment schedules. The first was a slow introductory regimen (low/ slow), starting with a dose of $0.625 \mathrm{mg} /$ day. Thereafter increments of $0.625 \mathrm{mg} /$ day were made at weekly intervals, until the dose reached $3.75 \mathrm{mg} /$ day. Then increments of 1.25 $\mathrm{mg} /$ day were prescribed at weekly intervals until the dose reached $25 \mathrm{mg} /$ day at 23 weeks. This dose was maintained until the end of the 26th week. The second was a rapid introductory regimen (high/fast), starting with a dose of 2.5 $\mathrm{mg} /$ day. Then increments of $2.5 \mathrm{mg} /$ day were prescribed at weekly intervals until the dose reached $15 \mathrm{mg} /$ day at six weeks. Thereafter increments of $5 \mathrm{mg} /$ day were prescribed at weekly intervals until the dose reached a maximum of 100 $\mathrm{mg}$ /day after 23 weeks. This dose was maintained until the end of the 26th week.

If patients reported an improvement so that they could cope with pretreatment work and social commitments and the total clinical rating score had dropped by $33 \%$, the dose was stabilised. If a $33 \%$ response was achieved but the patient continued to have functional disability, further increments were allowed.

The $33 \%$ figure was chosen because it is generally agreed that a $20 \%$ improvement can occur with placebo. The investigators agreed that this was a clinically significant improvement. When stable treatment had been reached patients were followed up at 3 monthly intervals.

Patients who encountered severe or intolerable side effects which did not respond to a reduction in dosage were taken off bromocriptine, recorded as treatment failures and where appropriate switched to a levodopa based treatment.

\section{Statistical methods}

To identify factors associated with dropping out due to severe side effects, logistic regression ${ }^{17}$ was used. A similar analysis was undertaken to detect prognostic factors associated with achieving the $33 \%$ improvement criteria.

In the analysis of amount of drug needed to achieve the improvement criteria, Cox's regression ${ }^{18} 19$ was applied because of the presence of censored observations (that is, those patients who had failed to reach $33 \%$ improvement at the end of 26 weeks.

Chi-square tests were used to assess the association between various variables of interest.
Table 2 Treatment groups at start of trial

\begin{tabular}{lcc}
\hline & Slow & Fast \\
\hline No of patients & 80 & 54 \\
No of patients analysed at 26 weeks & 76 & 53 \\
Mean age & 60.2 & 58.9 \\
Mean number of years since contracting & & \\
Parkinson's disease & 3.1 & 3.6 \\
Mean initial self care score & 24.7 & 26.4 \\
Mean initial Webster score & 60.9 & 66.0 \\
\hline
\end{tabular}

\section{Results}

Of the 134 patients entering the trial, one died from an unrelated cause and four patients were excluded from the analysis because they deviated from the protocol. One patient had received previous levodopa therapy, one patient had received glibenclamide, an oral antidiabetic agent which can be responsible for nausea, one patient had a history of psychosis, and one patient lived a distance from the investigator which made it impossible for him to attend regularly for clinical assessments at three weekly intervals. The results of the remaining 129 patients are presented. Seventy six patients were randomised to the slow treatment regimen and 53 to the fast. The clinical features of the treatment groups are shown in table 2.

No differences were found when the treatment groups were compared with respect to age; sex; presence or absence of a previous history of depression; other therapy; years since contracting Parkinson's disease; self rated score and initial Webster score.

Twenty one patients from the "high/fast" and 25 patients from the "low/slow" regimen were withdrawn from the study during the first 26 weeks for a variety of reasons (see table 3). Sixty five of the 129 patients $(50 \%)$ had reached the $33 \%$ improvement criterion by 26 weeks. These comprised 37 patients who followed the slow treatment regimen (49\%) and 28 patients who followed the fast treatment regimen $(53 \%)$. This difference is not statistically significant at the $5 \%$ level; as judged by the usual chi-square test (table 3). If, however, we compare the improvement rates of the two groups using only those 83 patients still in the trial at the end of 26 weeks, a different conclusion is reached; of the 51 patients in the slow group still in the trial at the end of 26 weeks, $37(73 \%)$, had reached the improvement criterion, in the fast group the corresponding figures are 32 and $28(88 \%)$. For these patients the difference between the proportion of responders in each group is significant $(p<0.05)$. Looked at conversely, the total number of nonresponders, who tolerated the drug, at 26 weeks was $24(31 \%)$-including 10 drop-outs because of non effect-in the slow group and six $(11 \%)$ in the fast group of which two were drop outs due to non effect. Again this difference is significant $(p<0.01)$. 
Table 3 Comparison of treatment groups at end of 26 weeks

\begin{tabular}{llc}
\hline & Slow & Fast \\
\hline Number of patients entered trial & 80 & 54 \\
Number of protocol deviants & 3 & 1 \\
Number died & 1 & 0 \\
Number of patients analysed at 26 weeks & 76 & 53 \\
$\begin{array}{l}\text { Number drop-outs due to side effects } \\
\text { Number drop-outs due to non-effect }\end{array}$ & $15(20 \%)$ & $19(36 \%)$ \\
$\begin{array}{l}\text { Number reaching 33\% improvement } \\
\text { criterion }\end{array}$ & $37(49 \%)$ & $28(53 \%)$ \\
$\begin{array}{l}\text { Number not reaching 33\% improvement } \\
\text { criterion }\end{array}$ & $14(18 \%)$ & $4(7 \%)$ \\
\hline
\end{tabular}

Logistic regression was again used in an attempt to identify any factors associated with a patient reaching the improvement criteria within the 26 weeks. The explanatory variables were as those used for the equivalent analysis of drop-outs. None of the explanatory variables was significantly associated with improvement.

The average daily doses of bromocriptine being taken by patients in the fast and slow group on reaching the $33 \%$ improvement criterion were 32.8 $\mathrm{mg} /$ day and $10.8 \mathrm{mg} /$ day respectively. The corresponding average times to reach the improvement criterion were 10 weeks and 11.6 weeks respectively. If compared by the usual $t$ test statistic (adjusted to account for the differing variances), a significant difference between the two groups is found for dose, but not for time. Such an analysis is, however, not applicable in this instance because it ignores the imbalances in the two groups of patients due to differences in proportions of patients dropping out due to severe side effects, and non-effect; similarly it ignores those patients who had not reached the improvement criterion at the end of 6 months. For such patients the only information available with regard to dose and time needed to reach the improvement criterion is that it is greater than the values of these quantities when either they dropped out or at the end of 26 weeks. Such censored observations can be allowed for by inclusion in a Cox's regression ${ }^{18}$ used to examine group differences in dosage and time at improvement; additionally the relationship of other explanatory variables to these two quantities could be examined in the same analysis.

One problem that needs to be considered is whether or not drop-outs due to severe side effects should be

Table 4 Estimate of dose and time (with approximate $95 \%$ Confidence Interval) needed to reach the improvement criterion in the two treatment groups allowing for the censored observations

\begin{tabular}{lll}
\hline & Estimate of dose (mg/day) & Estimate of time (weeks) \\
\hline Slow & $22 \cdot 0(14 \cdot 9-29 \cdot 1)$ & $22 \cdot 8(15 \cdot 5-30 \cdot 2)$ \\
Fast & $55 \cdot 4(33 \cdot 0-71 \cdot 8)$ & $14 \cdot 4(9 \cdot 1-19 \cdot 8)$ \\
\hline
\end{tabular}

counted as censored observations or simply removed from consideration altogether. Since our main concern is to draw inferences on dosage and time to improvement amongst a population of patients who do not suffer side effects, it seems more sensible simply to exclude those patients suffering severe side effects.

If this is done the results of a Cox's regression indicates group differences in both dose and time to improvement (the appropriate $\chi^{2}$ statistics are 9.51 d.f. $p<0.01$, and 5.91 d.f. $p<0.05$ ). None of the other explanatory variables were found to be associated with either dose or time to improvement.

Assuming that non responders at 6 months will ultimately reach criterion and that dose at improvement and time to reach improvement have an exponential distribution it is possible to theoretically estimate the values required to reach the improvement criterion in each group allowing for the censored observations. The results are shown in table 4 and indicate a marked difference in dose to reach improvement in the two groups, that in the slow being lower. For the time to improvement the results indicated that this is shorter in the fast treatment group. However, if a significant proportion of non responders at 6 months never subsequently reach criterion, then a more appropriate estimate for dose and time would lie between those given previously and those in table 4.

Patients dropped out of the trial either owing to the presence of severe side-effects (see table 5) or because they did not respond to the treatment. Severe sideeffects occurred in 34 patients ( $27 \%)$ and were multiple in 23 patients $(18 \%)$. Gastrointestinal disturbances occurred in 18 patients $(14 \%)$, predominantly nausea, vomiting, anorexia. Psychiatric disturbances occurred in 15 patients $(12 \%)$ [hallucinations $(5)$, confusion (4), depression (3), and anxiety (3)].

The duration of psychiatric side effects were short because in most cases, particularly at the beginning of

Table 5 Severe side-effects (34 patients) (Necessitating withdrawal from the study)

\begin{tabular}{llc}
\hline & Slow & Fast \\
\hline Psychiatric disturbances (ballucinations, & 10 & \\
confusion, depression, anxiety) & 8 & 5 \\
Gastro-intestinal & 5 & 10 \\
Postural hypotension & 4 & 2 \\
Visual disturbance & 3 & 1 \\
Headache & 1 & 0 \\
Mild dyskinesia* & 0 & 1 \\
Dyspnoea & 1 & 1 \\
Burning sensation of head and back & 1 & 0 \\
Hyperpigmentation of the hands & 1 & 0 \\
Paraesthesiae on the hands & 1 & 0 \\
Incontinence & & 0 \\
\hline Note: Some patients experienced multiple side-efiects necessitating \\
withdrawal. & & \\
*Concomitantly with psychiatric side effects.
\end{tabular}


treatment they were sufficiently severe to merit withdrawal. Psychiatric side effects became less common as the treatment period increased. Seven patients discontinued treatment because of postural hypotension, and a further five patients complained of visual disturbances not associated with postural hypotension.

Three patients experienced headaches sufficiently severe to necessitate withdrawal. Two patients, one from each group developed mild oro-buccal dyskinesia. Both were withdrawn from the trial because they concomitantly experienced psychiatric side effects. The patient from the slow group was male, aged 61 years, had been on treatment for 16 weeks and reached a dosage of $10 \mathrm{mg}$. The other patient was also male, aged 72 years, his treatment duration was 18 weeks and he had reached a dosage of $15 \mathrm{mg}$, this dosage having been limited because of his psychiatric side effects.

One patient each complained of dyspnoea, burning sensation of head and back, hyperpigmentation of hands, paraesthesiae in the hands and incontinence. Table 3 compares the two treatment groups with respect to the number of drop-outs at the end of 26 weeks.

A larger proportion of subjects in the fast group suffered severe side-effects (particular gastrointestinal side-effects) than in the slow group ( $36 \%$ compared to $20 \%$ ); the difference is significant at the $5 \%$ level as judged by the usual chi-square statistic. In the slow group 10 out of $76(13 \%)$ patients dropped out of the study with psychiatric disturbances. In the fast group five out of $53(9 \%)$ patients dropped out of the study with psychiatric side effects. This is not vastly different and would tend to suggest that those patients who experience psychiatric side effects on bromocriptine do so independently of dose or. rate of introduction to the drug.

The number of drop-outs due to non-effect was larger in the slow group than in the fast group (13\% compared with $4 \%$ ) but here the difference did not reach significance at the $5 \%$ level (table 3 ).

Further analyses of drop-outs was carried out using logistic regression. ${ }^{17}$ The explanatory variables considered in each analysis were age, sex, previous episode of depression, other medication, initial wADL, Initial Webster score, pre-treatment duration of Parkinson's disease and treatment group. A backward elimination procedure was used to eliminate those variables not associated with dropping out. The results of the analyses showed that the initial wADL was significantly associated with dropping out due to severe sideeffects at the $1 \%$ level.

Similar statistical analyses for those patients who dropped out due to non-response (10 in slow and two in fast group - table 3) showed no significant correlation with any factor.
Mild side effects were common especially those of the gastrointestinal tract. These were usually transient in nature. A greater proportion of patients in the fast treatment group suffered mild side effects.

\section{Discussion}

The study confirms that bromocriptine is an effective treatment in previously untreated patients with Parkinson's disease $e^{101213}$ half of the patients obtaining a $33 \%$ improvement in clinical rating compared with their pretreatment values. Teychenne ${ }^{11}$ suggested that lower doses of bromocriptine than had been previously recommended ${ }^{7-10}$ introduced slowly gave an initial beneficial response without patients experiencing side effects normally observed by introducing the drug using a faster dosage regimen. In this study the estimated time to reach the improvement criterion in the slow group (22.8 weeks) was longer than the corresponding estimate for the fast group (14.4 weeks); for dose to improvement the reverse is found with the estimate for the slow group $(22.0 \mathrm{mg} /$ day $)$ being lower than that for the fast group $(55.4 \mathrm{mg} /$ day $)$.

In contrast to the publication some time ago by Teychenne ${ }^{11}$ and the more recent one by Olanow ${ }^{15}$ our results confirm the preliminary report by Pearce and Pearce $^{14}$ that the minimum effective dose of bromocriptine in most previously untreated Parkinsonian patients is in excess of $20 \mathrm{mg} /$ day using a slow introductory regimen. Whereas these previous studies have been carried out in a small series of patients the size of our series ( 80 in the slow group) adds weight to this conclusion. If a fast introductory regimen is used the dose of bromocriptine required is higher $(55.4 \mathrm{mg} /$ day). The reason for this difference is unexplained.

The use of doses of bromocriptine of $20-40 \mathrm{mg} /$ day with a faster build up time between that used in the slow and fast regimens might therefore be the ideal way of giving bromocriptine to de novo patients. There could also be potential long term benefits in employing a minimum effective dose to treat Parkinsonian patients so as to avoid future complications of treatment and to postpone or perhaps prevent failure to control symptoms of the disease. Bromocriptine, which has been under clinical investigation for over 10 years, as primary therapy, has not been reported to produce on-off oscillations and dyskinesia is currently rare..$^{8-10}$ The possibility of using a combination of small doses of a levodopa preparation and bromocriptine together "de novo" has been suggested. ${ }^{613}$ Other controlled prospective studies are required to investigate this further. The incidence of drop-outs due to severe side effects was greater in the "high/fast" regimen (36\% compared to $20 \%$ ) the difference being significant at the $5 \%$ level. Drop-outs due to non-effect were also higher in the "low/slow" group than in the 
"high/fast" group ( $12 \%$ compared with $4 \%$ ), but this did not reach statistical significance at the $5 \%$ level. The only factor found to be associated with dropping out due to severe side effects was a high initial wADL. This result is of interest but is so far unexplained. Factors such as age, sex, years since contracting Parkinson's disease, past history of depression, initial Webster scores had no effect on the chance of reaching the improvement criteria, dropping out due to severe side effects or the dose of drug needed to reach the improvement criteria.

The most common mild side effects were those of the gastrointestinal tract and were transient in nature. These can be reduced by domperidone, a peripherally acting dopamine antagonist. ${ }^{21}$

The incidence of psychiatric side effects reported in this trial is higher than would be expected in a similar group of patients treated with levodopa therapy. The level of psychiatric side effects is, however, comparable with those experienced in other series of patients receiving bromocriptine alone. Of the 28 patients treated by Lees and Stern' for one year, four subsequently withdrew because of psychiatric side effects (two toxic confusion, two visual hallucinations). $\mathrm{Rascol}^{8}$ reported one case of acute confusion necessitating withdrawal on a dose of $\mathbf{4 0} \mathrm{mg}$ daily. Rinne ${ }^{6}$ also reported hallucinations in five of 76 patients in his trial: an incidence of $7 \%$. In a small open investigation by Pearce and Pearce of slow and fast introductory regimens of bromocriptine, two patients out of 10 withdrew because of severe psychiatric reactions. Both were from the fast group.

The number of patients achieving a worthwhile therapeutic response to bromocriptine is less than one might predict in a comparable group given levodopa. If it can be demonstrated with long term follow up that bromocriptine therapy has fewer side effects this would have to be balanced against the less favourable initial results.

It is intended to follow patients for up to 5 years. In the long term follow-up patients who either did not achieve the $33 \%$ improvement criterion, or who were non responders, or who dropped out due to severe side effects were switched to levodopa based treatment. This longer term evaluation should help to establish if it is beneficial to use the minimum effective dose of bromocriptine to prevent complications of long term therapy while maintaining effective control of symptoms.

We thank Dr A J Munro, Medical Director, The
Association for Clinical Research and Mrs E R Baister of Sandoz Products Limited (UK) for their invaluable help in the design of the protocol and co-ordination of the study respectively.

\section{References}

1 Calne DB, Teychenne PF, Claveria LE, Eastman R, Greenacre JK. Bromocriptine in Parkinsonism. Br Med J 1974;4:442-4.

2 Lieberman AN, Gopinathan G, Hassouri H, Neophytides A, Goldstein M. Should dopamine agonists be given early? A review of nine years experience with bromocriptine. Can $J$ Neurol Sci 1984;11:233-7.

3 Grimes JD, Kind DB, Kofman OS, Molina-Negro P, Wilson AF, Bouchard S. Bromocriptine in the management of end of dose deterioration in Parkinson's disease. Can J Neurol Sci 1984; 11:452-6.

4 Hoehn MMM, Elton RL. Low dosages of bromocriptine added to levodopa in Parkinson's disease. Neurology 1985;35:199-206.

5 Pfeiffer RF, Wilken K, Glaeske C, Lorenzo AS. Low-dose bromocriptine in Parkinson's disease. Arch Neurol 1985;42: 586-8.

6 Rinne UK. Combined bromocriptine-levodopa therapy early in Parkinson's disease. Neurology 1985;35:1196-8.

7 Parkes JD, Marsden CD, Donaldson I, et al. Bromocriptine treatment in Parkinson's disease. J Neurol Neurosurg Psychiatry 1976;39:184-93.

8 Rascol A, Guiraud B, Montstruc JL, David J, Clanet MJ. Longterm treatment of Parkinson's disease with bromocriptine. J Neurol Neurosurg Psychiatry 1979;42:143-50.

9 Lees AJ, Stern GM. Sustained bromocriptine therapy in previously untreated patients with Parkinson's disease. J Neurol Neurosurg Psychiatry 1981;44:1020-3.

10 Stern GM, Lees AJ. Sustained bromocriptine therapy in 50 previously untreated patients with Parkinson's disease. Adv Neurol 1983;37:17-21.

11 Teychenne PF, Bergsrud D, Racy A, Vern B. Low-dose bromocriptine therapy in Parkinson's disease. Res Clin Forums 1981; 3:37-53.

12 Pearce I, Pearce JMS. Bromocriptine in Parkinsonism. Br Med J 1978;1:1402-4.

13 Grimes JD, Delgado MR. Bromocriptine: Problems with low-dose de novo therapy in Parkinson's disease. Clin Neuropharmacol 1985;8:73-7.

14 Pearce I, Pearce JMS. Low-dose bromocriptine in Parkinson's disease. J Neurol Neurosurg Psychiatry 1985;48:388-9.

15 Olanow CW, Alberts MJ. Low-dose bromocriptine in previously untreated Parkinson patients. In: Fahn S, Marsden CD, Jenner $\mathrm{P}$, Teychenne P, eds. Approaches to the use of Bromocriptine in Parkinson's disease. Raven Health Care Communications, 1985:15-23.

16 Webster DD. Critical analysis of the disability of Parkinson's disease. Mod Treat 1968;5:257-82

17 Lee ET. Statistical methods for survival data analysis. Belmont: Lifetime Learning Publication, 1980:122.

18 Cox DR. Analysis of Binary Data. New Edition. London: Chapman and Hall, 1979.

19 Cox DR. Regression models and life tables. J R Stat Soc, Series $B$ 1972;34:187-220.

20 Pocock SI. Allocation of patients to treatment in clinical trials. Biometrics 1979;35:183-97.

21 Quinn N. Bromocriptine and domperidone in the treatment of Parkinson's disease. Neurology 1981;31:662-7. 